\title{
Photostability Drug Study
}

National Cancer Institute

\section{Source}

National Cancer Institute. Photostability Drug Study. NCI Thesaurus. Code C96087.

A study conducted to determine the extent of effect that exposure to light has on a pharmaceutical product. 